Relations industrielles

Industrial Relations

\title{
The Nature of Price Theory, by H.H. Liebhapsky, Revised Edition, The Dorsey Press, Homewood. Illinois, August 1968, 643 pages.
}

\section{Jacques St-Laurent}

\section{Volume 24, numéro 1, 1969}

URI : https://id.erudit.org/iderudit/028014ar

DOI : https://doi.org/10.7202/028014ar

Aller au sommaire du numéro

Éditeur(s)

Département des relations industrielles de l'Université Laval

ISSN

0034-379X (imprimé)

1703-8138 (numérique)

Découvrir la revue

Citer ce compte rendu

St-Laurent, J. (1969). Compte rendu de [The Nature of Price Theory, by H.H. Liebhapsky, Revised Edition, The Dorsey Press, Homewood. Illinois, August 1968, 643 pages.] Relations industrielles / Industrial Relations, 24(1), 230-231. https://doi.org/10.7202/028014ar

Tous droits réservés (C Département des relations industrielles de l'Université Laval, 1969
Ce document est protégé par la loi sur le droit d'auteur. L’utilisation des services d’Érudit (y compris la reproduction) est assujettie à sa politique d'utilisation que vous pouvez consulter en ligne.

https://apropos.erudit.org/fr/usagers/politique-dutilisation/ 
tera pour l'organisation aucun progrès sensible: le vainqueur détient le championnat jusqu'au match revanche. Des efforts sont quotidiennement faits pour que la prise de décision soit un mécanisme finement rationnel. Trois étapes constituent essentiellement le processus de solution des problèmes: la perception, la recherche de solutions originales et l'évaluation de ces solutions. Les caractéristiques du problème et du chercheur vont facilement influencer les résultats de chacune de ces étapes d'où la difficulté d'éliminer complètement le biais dû aux attitudes de base et aux motivations de ceux qui affrontent ces problèmes.

\section{Hervé GAUTHIER}

Peach Ceri, West Indian Migration to Britain, Oxford University Press, London 1968.

Le travail présenté dans cet ouvrage est un essai d'analyse et d'interprétation des mouvements migratoires entre les Indes Occidentales et la Grande-Bretagne.

L'analyse se fonde sur des statistiques récentes mais incomplètes. Elle tente de décrire globalement et quantitativement les groupes d'émigrants, de définir leur destination exacte et de retrouver les lieux de leur installation. L'analyse conduit à une interprétation des causes et conséquences de ces mouvements migratoires. Les causes sont simples et peu définies; elles ne permettent donc pas l'élaboration d'un modèle d'analyse pour d'autres situations de même genre. Les conséquences sont étudiées trop rapidement et par rapport à une situation trop particulière; elles empêchent donc de prévoir, sur une échelle universelle, les suites de phénomènes semblables.

En somme, cet ouvrage présente un intérêt certain, mais très particulier. Il n'invite pas à des études plus approfondies et ne permet pas une théorisation certes nécessaire dans ce domaine.

\section{René PARENTEAU}

Essays in Labour History, by Asa Briggs and John Saville, Papermac 191, Macmillan 1967, 364 pages.

Cet ouvrage a été préparé en collaboration et il est dédié à la mémoire de ce grand historien du travail que fut
G.D.H. Cole, professeur à Oxford en son temps, et qui pourrait être aussi bien cité comme économiste que sociologue ou politicologue.

Les éditeurs présentent d'abord un certain nombre de textes à caractère biographique rédigés par des personnes qui ont bien connu le professeur.

Mais l'essentiel de l'ouvrage est constitué de neuf essais en histoire du travail sous la signature d'autant d'historiens qui d'une façon ou d'une autre ont suivi les traces du maître. L'ensemble de ces essais présente un vaste tableau de l'histoire du travail, notamment en Grande-Bretagne, au cours du dix-neuvième siècle.

Il $\mathrm{y}$ est question tout aussi bien $\mathrm{du}$ concept de classe du début de ce siècle que de la participation de certains éléments du mouvement ouvrier anglais à la première Internationale.

On y trouve aussi des textes sur les circonstances qui ont entouré l'affaire Taff Vale, sur les conditions de travail en terme d'usages, de salaires et de charge de travail au XIXe siècle, et quelques autres.

Briggs et Saville mettent ainsi un précieux instrument de travail à la disposition de ceux qui s'intéressent à cette période importante de l'histoire du travail en Grande-Bretagne.

\section{Jean BERNIER}

The Nature of Price Theory, by H.H. Liebhapsky, Revised Edition, The Dorsey Press, Homewood. Illinois, August 1968, 643 pages.

Malgré les prétentions de l'auteur à l'effet que la lecture de son excellent manuel exige du lecteur qu'il sache seulement additionner, soustraire, multiplier et diviser, il faut bien avouer que son livre ne se lit pas comme un roman-policier et qu'il exige davantage.

Ecrit surtout à l'intention des étudiants sous-gradués, il suppose de la part du lecteur, sinon un entraînement mathématique minimum, du moins un certain esprit mathématique et une certaine habileté à traduire dans ce langage et celui de la géométrie les relations fondamentales que l'on peut abstraire de la réalité économique. 
Un étudiant sérieux possédant cet esprit et cette habileté trouvera dans l'étude de ce manuel beaucoup de plaisir et de satisfaction et ce, pour plusieurs raisons.

Tout d'abord, bien que de façon concise mais très rigoureuse, l'auteur prend le temps, au tout début de son manuel, d'expliquer à fond un grand nombre de concepts de base. C'est ainsi qu'on y explique, entr'autres, des concepts élémentaires comme ceux des quantités marginales, de la droite, des taux de changement etc; puis des concepts plus compliqués comme ceux de la dérivée, des lignes de contour, des logarithmes dans la base c, de l'utilité marginale etc.

On notera que l'auteur prend le temps, ce qui n'est pas habituel dans les manuels de ce genre, de revenir sur des notions de base qui ne relèvent pas strictement du domaine de l'analyse économique comme le droite et les logarithmes. Cela donne l'avantage de partir sur un pied solide, sans comporter pour cela le désavantage de trop retarder l'analyse économique parce que l'auteur ne donne que l'essentiel de ces choses.

En second lieu, l'auteur fournit suffisamment d'informations, soit dans les graphiques, soit dans les tableaux, pour que le lecteur puisse lui-même tracer ses propres graphiques à l'échelle qui lui plait lui donnant ainsi l'occasion d'apprendre « en faisant ». De plus, ces informations comportent l'avantage supplémentaire de rendre concrètes des choses déjà suffisamment abstraites en « chiffrant 》 les variables et les constantes des équations.

Enfin, dans de nombreux cas, l'auteur rappelle le résultat des études empiriques tentées dans le but de vérifier dans quelle mesure la théorie des prix est conforme à la réalité. Cela lui permet, avec beaucoup de justesse, de souligner les limitations de la première mais aussi son évidente utilité, avec la conséquence que l'intérêt de l'étudiant envers la théorie des prix ne peut que croître puisqu'il en voit des applications.

Voilà pour l'ensemble des caractéristiques qui permettent de faire de ce manuel un manuel à part.

Quant au reste, il faut dire que l'auteur aborde la matière habituellement couverte par les manuels de ce genre, soit la théorie de la consommation, celle de la production et celle de la distribution. Dans ce dernier cas, l'exposé sur l'intérêt, les profits et la rente est plus succinc que d'habitude, ce qui soulage le lecteur des discussions et distinctions oiseuses de certains manuels tout en lui permettant de saisir l'essentiel du problème.

A cela, il ajoute les considérations sur la relativité du bien-être économique impliqué par la théorie statique des prix et sur la programmation linéaire comme technique mathématique permettant d'approximer pour des problèmes concrets la solution théorique prévue par l'utilisation des courbes d'indifférence d'échelle et de budget.

Enfin, voilà un manuel qui développe les éléments de la théorie statique des prix avec beaucoup de soin, beaucoup de rigueur et un souci constant d'en assurer l'intégration. C'est un manuel qui en est à sa deuxième édition. Sa qualité laisse croire qu'il y en aura d'autres.

\section{Jacques ST-LAURENT}

Histoire et politique du droit du travail, par P. Virton, s.j., Bibliothèque de la recherche sociale, Spes, Paris, 1968, 254 pages.

S'il existe de nombreux manuels et traités qui ont pour objet de présenter le droit français du travail dans la totalité de ses techniques juridiques, plus rares sont les ouvrages consacrés à l'histoire du droit du travail, comme tel.

Celui de Virton veut justement tenter de replacer dans leur contexte historique et politique les grandes tendances actuelles de ce droit particulier et de montrer comment il s'est développé à la suite de la naissance de relations sociales nouvelles ou de la transformation de relations antérieures et sous la pression de mouvements d'opinion suffisamment forts pour amener le pouvoir politique à modifier les institutions juridiques.

Nous sommes donc ainsi amenés à considérer dans leur évolution des relations qui naissent du travail subordonné et le droit qui les régit depuis la naissance du prolétariat d'industrie jusqu'à l'époque actuelle de la détermination collective des conditions de travail et de la participation des salariés à la vie économique et sociale. 